\title{
Existence of Phase Explosion during Laser Ablation and its Effects on Inductively Coupled Plasma - Mass Spectroscopy
}

\author{
Jong H. Yoo, Oleg V. Borisov, Xianglei Mao, and Richard E. Russo* \\ Lawrence Berkeley National Laboratory \\ Berkeley, California 94720, U.S.A
}

\begin{abstract}
A sudden increase in crater depth was observed during high irradiance $\left(>10^{10}\right.$ $\mathrm{W} / \mathrm{cm}^{2}$ ) laser ablation of silicon, and it is attributed to the phenomena of phase explosion. The threshold irradiance for phase explosion showed a dependence on two laser parameters; laser beam spot size and wavelength. For a larger beam size and longer incident wavelength, a higher laser irradiance was required to generate phase explosion. The rapid increase of crater depth above the phase explosion threshold irradiance correlated with a significant increase in the ICP-MS signal intensity. The ratio of crater volume to ICP-MS intensity, which represents entrainment efficiency, remained the lowest at laser irradiances slightly above the phase explosion threshold. However, this ratio increased at irradiances well above the threshold $\left(>10^{11} \mathrm{~W} / \mathrm{cm}^{2}\right)$. Chemical analysis using laser ablation at irradiance above $10^{11} \mathrm{~W} / \mathrm{cm}^{2}$ provides increased sensitivity via improved entrainment and transport efficiency and increased ablation rate.
\end{abstract}

\footnotetext{
* To whom the correspondence should be addressed. E-mail: RERUSSO@lbl.gov, Fax: 510-486-7303
} 


\section{INTRODUCTION}

Laser ablation with inductively coupled plasma (ICP), mass spectroscopy (MS), or atomic emission spectroscopy (AES) has been investigated extensively for direct solid sample chemical analysis due to its advantages: any sample material, minimal sample preparation, localized (spatial) analysis, and minimal exposure of personnel to toxic samples $^{1-11}$. In many experimental systems, the solid sample is ablated inside a simple chamber and the ablated mass is transported by a carrier gas to the ICP for analysis by AES or MS. In the ideal case, the chemical composition of the ablated mass would be identical to the parent sample to ensure accuracy, and transport efficiency would be maximal to enhance sensitivity. However, sampling conditions often deviate from ideal due to factors such as preferential vaporization of elements from the sample, failure of large particulates to be transported, and fractionation caused by incomplete vaporization of large particulates in the $\mathrm{ICP}^{12-14}$. For these reasons, the quantity of the ablated mass and its size distribution are crucial factors that must be known in order to optimize the laser parameters for samples of interest.

Irradiance is a laser parameter that can significantly influence the amount of ablated mass and the particle size distribution ${ }^{1,14}$. Depending on the laser irradiance and the sample material, mass removal can occur through desorption, thermal evaporation, surface instability of molten mass, exfoliation, phase explosion, and other mechanisms. The mass ablation rate follows a power law with laser irradiance (I) as $\mathrm{I}^{\mathrm{n}}$. In a low irradiance region $\left(<3 \times 10^{8} \mathrm{~W} / \mathrm{cm}^{2}\right.$ for a ns pulsed laser $)$, desorption and thermal evaporation may dominate sample removal processes ${ }^{15,16}$, and the index $\mathrm{n}$ is greater than

one. A non-thermal mechanism occurs with increasing laser irradiance ${ }^{16}\left(>3 \times 10^{8} \mathrm{~W} / \mathrm{cm}^{2}\right.$ 
for ns laser) and the index $\mathrm{n}$ is less than one. When laser irradiance is increased further (> $10^{10} \mathrm{~W} / \mathrm{cm}^{2}$ ), a sudden increase in mass removal occurs, and $\mathrm{n}$ becomes greater than one $^{16}$. The increase in mass removal is attributed to the onset of phase explosion ${ }^{17}$. Phase explosion is a rapid boiling process due to homogeneous nucleation in a superheated volume of liquid near its critical state. When a high irradiance, short $(<10$ nsec) pulse laser beam is focused on a sample surface, a volume of material is rapidly melted; the molten mass can be heated beyond its thermodynamic boiling temperature. Density fluctuations in the superheated molten pool can lead to bubble formation. Thermodynamically, bubbles with radius larger than a critical value, $r_{\mathfrak{c}}$, can grow spontaneously while bubbles with sizes less than $r_{c}$ are likely to collapse. Heat transfer from the superheated surrounding to the bubble interface enables bubble growth. Bubbles generated throughout the superheated volume that grow to $r_{c}$ will expand instantaneously. The rapidly expanding bubbles exert pressure within the volume leading to the ejection of mass. This process is known as explosive boiling or phase explosion. Yoo et al showed that for the ablation of bulk silicon, irradiance on the order of $10^{10}$ $\mathrm{W} / \mathrm{cm}^{2}$ was required to generate phase explosion ${ }^{18}$.

The particle size distribution of the ablated mass depends on the ablation mechanism. The ablated mass may be in the form of atomic-sized vapor if desorption or thermal evaporation dominate, whereas it may be in the form of micron-sized particulates when liquid droplet ejection due to surface instability of molten mass occurs. Mass removal can also occur in the form of bulk solid flakes for an exfoliation process ${ }^{14}$, and a mixture of vapor and large particulates from a phase explosion process ${ }^{17,18}$. 
The significant increase in ablated mass quantity and generation of large particulates due to phase explosion will impact transport efficiency and ICP-AES or MS signal intensity. To the best of our knowledge, there have not been any studies that investigated the effect of phase explosion on transport efficiency or ICP-MS and AES signal intensity. In this work, crater depths were measured below and above the phase explosion threshold to show the significant increase in mass removal once phase explosion occurs. Above the phase explosion threshold, large particulate ejection was observed by shadowgraph images. The dependence of the threshold irradiance for phase explosion on various laser parameters such as the beam spot area and wavelength was investigated. A correlation of crater depth and volume data with the ICP-MS signal intensity was made over the irradiance range in which phase explosion occurred. The sudden increase in crater depth at the phase explosion threshold irradiance is seen in the ICP-MS integrated intensity. The ratio of ICP-MS integrated intensity to net crater volume (volume below the original surface subtracted by the volume above) was estimated over the irradiance range in which phase explosion occured. Crater volume is a close estimation of mass removed from the sample, whereas the integrated ICP-MS signal over the sampling time is proportional to the mass reaching the ICP. The behavior of this ratio over the investigated irradiance range shows how the entrainment and transport efficiency of the ablated mass vary before and after phase explosion.

\section{EXPERIMENTAL SECTION}

The experimental system for laser ablation sampling includes an ICP-MS (VG Elemental, PQ3), a 3 ns Nd:YAG laser (Coherent, Infinity), and an ablation cell. The 
laser beam spatial power profile is close to Gaussing. Si samples were used in this work. Ablated mass was transported to the ICP torch along a 1.4 m polyethelene tube with 4.1 $\mathrm{mm}$ i.d. The ICP-MS was operated at forward RF power of $1350 \mathrm{~W}$ and gas flow rates of $14,1.0$, and $1.0 \mathrm{~L} \mathrm{~min}^{-1}$ for plasma, auxiliary, and carrier flows, respectively. The ICPMS lens voltages were optimized to achieve maximum sensitivity for ${ }^{29} \mathrm{Si}$. The ICP-MS was operated in a single-ion-monitoring mode. Time-resolved ICP-MS signal intensity for ${ }^{29} \mathrm{Si}$ was measured during single pulse laser ablation of the sample, with a dwell time of $12 \mathrm{~ms}$ and 3 points per peak. Depending on the laser irradiance it took about $20-30 \mathrm{~s}$ for the signal intensity to return to the background level. The laser pulse was fired following a delay of $3 \mathrm{sec}$ after starting the ICP-MS data acquisition. Intensity data recorded during the time delay were used for background correction. The integrated intensity reported herein is defined as the ICP-MS ${ }^{29} \mathrm{Si}$ intensity integrated over the acquisition time after background subtraction.

The morphology, depth, and volume of the craters were measured with a white light interferometric microscope (Zygo, NewView 200). The shadowgraph-imaging experimental setup for observing particulate ejection from the ablated surface is shown in figure 1. A Nd:YAG laser (Coherent, Infinity) was used as the ablation laser. A Continuum YG601 Nd-YAG laser $(\mathrm{FWHM}=35 \mathrm{ps}, \lambda=532 \mathrm{~nm})$ was used as a probe beam. The probe beam was aligned parallel to the silicon surface and perpendicular to the ablation beam, and directed to a CCD camera (Photometrics Model CH250/A). The time delay between the ablation pulse and the probe pulse, which ranged from 5 ns to 30 $\mu$ sec, was controlled by using a four-channel digital delay/ pulse generator (Stanford Research Systems, Model DG535). 


\section{RESULTS AND DISCUSSION}

\section{a) Phase explosion}

Phase explosion occurs when the sample is rapidly heated beyond its normal boiling temperature and becomes a metastable liquid near its critical state. While in this state, slight perturbation in density can lead to the initiation of vapor nuclei in the superheated liquid, which was known as homogeneous nucleation. Once these vapor bubbles reach a critical size, further growth is spontaneous and the superheated volume explodes $^{17-19}$. A dramatic change in the crater depth observed at different laser irradiances is an indication of phase explosion (Figure 2). These data show the crater cross section profiles in silicon at two laser irradiances; one slightly below the threshold $\left(20 \mathrm{GW} / \mathrm{cm}^{2}\right)$ and one slightly above $\left(24 \mathrm{GW} / \mathrm{cm}^{2}\right)$. Each crater was produced by using one laser pulse. For the $3 \mathrm{~ns}$ laser pulse with wavelength of $266 \mathrm{~nm}$ and spot size of $35 \mu \mathrm{m}$, the threshold irradiance was approximately $22 \mathrm{GW} / \mathrm{cm}^{2}$. Below the threshold irradiance, the crater was characterized by a smooth bottom and a rim that surrounded a thermally affected area. Just above the threshold, the crater was significantly deeper with rough bottom and minimal surface rim. Once phase explosion occurred, there was a significant increase in the quantity of mass removed, as evidenced by a more than four fold increase in the crater depth (note y-axis scale change); the crater depth changed dramatically to approximately $7 \mu \mathrm{m}$.

Particulate ejection resulting from phase explosion of the surface was observed by shadowgraph imaging when the laser irradiance was greater than the threshold (22 
$\mathrm{GW} / \mathrm{cm}^{2}$ ); below the threshold particulate ejection was not observed. However, in the lower laser irradiance region, sub-micron particles do exist but cannot be detected by the imaging system. Sub-micron particles result from splashing of the molten liquid by plasma recoil and condensation of vapor. Figure 3 shows time-resolved shadowgraph images of the mass ejection at $39 \mathrm{GW} / \mathrm{cm}^{2}$, which is above the phase explosion threshold for the $35 \mu \mathrm{m}$ beam size. Shock wave propagation is observed shortly after the laser pulse. After the shock wave passes, there is a time period lasting $\sim 300-400 \mathrm{nsec}$ where mass removal was not observed. Approximately $400 \mathrm{nsec}$ after the laser pulse, significant mass was ejected from the silicon surface. The ejection of these particulates lasts for about $30 \mu \mathrm{sec}$. A precise determination of the ejected particulate sizes is difficult due to the presence of diffraction rings around the particles in the images. Larger particles will have fewer diffraction rings and a sharper boundary. The size of the large particles is estimated to be approximately 10 microns. In these images, there are approximately 10 particles in the space of $400 \mu \mathrm{m} \times 500 \mu \mathrm{m}$. The volume of these particles is approximately $4000 \mu \mathrm{m}^{3}$. The volume of the crater from this irradiance was approximatley $6000 \mu \mathrm{m}^{3}$. Considering that some particles are left on the surface and small particles cannot be measured, the data suggest that the deep crater was mainly formed by the ejection of the larger size $(\sim 10 \mu \mathrm{m})$ particles.

The threshold of crater depth at $22 \mathrm{GW} / \mathrm{cm}^{2}$ was not caused by the ignition of the plasma due to the inverse Bremsstrahlung absorption. An estimate of the irradiance threshold $\Phi_{t h r}$ for plasma ignition is given by the relationship ${ }^{20}: \Phi_{t h r}(\sqrt{\tau}) \geq B$, where $\tau$ is the laser pulse duration and, $B \approx 4 \times 10^{4} \mathrm{~W} \cdot \mathrm{s}^{1 / 2} \cdot \mathrm{cm}^{-2}$ for $\tau$ in the range of millisecond to nanosecond. In this experiment, the laser pulse duration was $3 \mathrm{~ns}$; 
therefore, the estimated threshold for plasma ignition is : $\Phi_{t h r} \approx 7 \times 10^{8} \mathrm{~W} / \mathrm{cm}^{2}$. The plasma ignition threshold is almost two orders of magnitude lower than the threshold observed in this work. After plasma ignition, an approximate, material-independent relation between ablation depth, plasma temperature, and electron number density with laser irradiance can be derived based on plasma heated by inverse bremsstrahlung absorption $^{21}$. The measured ablation depth, plasma temperature, and electron number density versus laser irradiance are in good agreement with the theoretical model ${ }^{22}$. The agreement of experiment and theory indicates the plasma ignition occurs at an irradiance below the threshold observed in this work. Balazs et. al. ${ }^{23}$ calculated melt depth to be 2 $\mu \mathrm{m}$ at $1.2 \mathrm{GW} / \mathrm{cm}^{2}$. Splashing of molten material due to the vapor recoil also occurs in the lower irradiance region. But, splashing of molten material due to recoil pressure cannot explain the sudden change in crater depth. For laser irradiances above the threshold, the observed crater characteristics were attributed to phase explosion (or explosive boiling) in conjunction with induced-transparency of the liquid silicon near the thermodynamic critical temperature. A detailed theoretical phase explosion model can be found in reference [18], which demonstrates a good agreement between theory and experimental results.

The existence of phase explosion can have several important effects for laser ablation solid sampling. Obviously, the quantity of mass is significantly increased which will influence sensitivity. Also, a change in the size distribution of the ejected mass occured, which will influence entrainment and transport efficiency to the ICP. Finally, phase explosion may be a region in which the ablated volume is independent of the 
material properties. If this is the case, quantitative analysis would be possible using nonmatrix matched standards. Also fractionation may be relatively less. These later two effects will be investigated in future research.

\section{b) Phase explosion threshold}

The effects of different laser conditions on the threshold irradiance for phase explosion were investigated in this work. Single-pulse ablation of the single crystal silicon sample was sudied by varying three parameters; laser irradiance $\left(10^{9} \sim 10^{12}\right.$ $\left.\mathrm{W} / \mathrm{cm}^{2}\right)$, beam spot size $(18 \sim 70 \mu \mathrm{m})$, and wavelength $(\lambda=266,532$, and $1064 \mathrm{~nm})$. The threshold irradiance was found to be dependent on the laser wavelength and the beam spot size. The ablated crater depths as a fuction of laser irradiance are plotted for three wavlengths in Figure 4. For the $35 \mu \mathrm{m}$ laser beam diameter, the $266 \mathrm{~nm}$ induced phase explosion at an irradiance of $\sim 22 \mathrm{GW} / \mathrm{cm}^{2}$, followed by $532 \mathrm{~nm}$ at an irradiance of $\sim 420$ $\mathrm{GW} / \mathrm{cm}^{2}$ (roughly one order of magnitude higher). At $1064 \mathrm{~nm}$, phase explosion was not observed over the available irradiance range of these studies.

Stronger plasma shielding and deeper optical penetration depth as the wavelength increases can be resposible for the change in phase explosion threshold. Plasma shielding reduces the quantity of laser energy available for heating the sample and the larger optical penetration depth leads to volumetric heating of the sample; a higher irradiance is requied to heat the irradiated volume to its critical state. The ablated mass, which contains both ionized and neutral species of atoms and molecules, formed by the early part of laser pulse, can interact with the latter part of the pulse, effectively absorbing the laser energy and shielding the target from the incident radiation. Liu et. al. showed that for UV laser irradiances significantly below the phase explosion threshold, the plasma was sufficiently 
ionized; electron-ion inverse bremsstrahlung may dominate absorption for UV radiation $^{22}$. The absorption coefficient $\alpha_{\mathrm{p}}\left(\mathrm{cm}^{-1}\right)$ for electron-ion inverse bremsstrahlung is proportional to $n_{i}^{2} \lambda^{2} / T^{3 / 2}$ where $n_{i}, \mathrm{~T}$, and $\lambda$ are ion density, temperature of the plasma, and the incident light wavelength ${ }^{22}$. Therefore, at longer wavelengths, substantially higher absorption of the incident radiation can occur by the plasma and less radiation energy will be transmitted to the sample surface ${ }^{24-26}$.

Another factor that could contribute to a higher threshold irradiance at longer wavelengths is the wavelength-dependent optical penetration depth in silicon. At 266 $\mathrm{nm}$, the optical penetration depth is on the order of ten nanometers; the incident optical energy is absorbed in a very small region near the surface, rapidly increasing its local temperature. At $532 \mathrm{~nm}$, the optical penetration depth increases to hundreds of nanometers, which is still an order of magnitude less than the thermal diffusion length $(\sim$ $1 \mu \mathrm{m}$ ) for the $3 \mathrm{~ns}$ laser pulse. Therefore, as with the $266 \mathrm{~nm}$ radiation, the optical energy is deposited in a relatively shallow region near the surface and dissipated primarily thorugh heat conduction into the bulk sample. At $1064 \mathrm{~nm}$, the optical penetration depth $(\sim 200 \mu \mathrm{m})$ is two orders of magnitude greater than the thermal diffusion length; the absorption of the incident radiation and subsequent heating will be volumetric. Since heating occurs over a substantially larger volume, the temperature will be less than at 266 and $532 \mathrm{~nm}$. At $1064 \mathrm{~nm}$, strong plasma absorption and volumetric heating may prevent the sample from attaining the superheated liquid state for the duration required for vapor nuclei to grow to the critical size.

The threshold irradiance for phase explosion was found to be dependent on the laser beam spot size. Figure 5 shows the dependence of the threshold on the laser beam 
sizes for $\lambda=266 \mathrm{~nm}$ and $532 \mathrm{~nm}$. For both 266 and $532 \mathrm{~nm}$, the threshold irradiance increased for a larger beam diameter. The dependence of phase explosion threshold irradiance on the laser beam size can be explained in terms of plasma expansion dynamics. For small beam sizes, the expansion of the plume is spherical, occurring in three dimensions during the laser pulse. When the beam diameter is small, a three dimensional expansion of the plasma occurs during the laser pulse ${ }^{27}$. A reduction in the plasma expansion dimension for larger beam diameters will result in a decrease in the effective volume of the plasma layer, and hence higher ion/electron number density. Increased ion/electron density increases the plasma absorption of incident radiation; therefore, a higher irradiance will be required to initiate phase explosion.

\section{c) Correlation of crater depth with ICP-MS intensity}

The MS signal intensity is proportional to total mass removed from the sample and

transported to the ICP ${ }^{28,29}$. For constant ICP conditions, the change in the relationship (slope) of ICP-MS intensity versus laser irradiance can signify a change in the ablation and transport processes. At the onset of phase explosion, there is a dramatic increase in both crater depth and volume; the enhanced mass removal should result in a rapid rise in the ICP-MS integrated signal intensity. Figure 6 shows the ICP-MS integrated intensity and crater depth as a function of laser irradiance for the silicon sample ablated using a 45 $\mu \mathrm{m}$ beam diameter spot size. A sharp increase, and subsequent saturation in the crater depth were observed with the threshold at approximately $5 \times 10^{10} \mathrm{~W} / \mathrm{cm}^{2}$. Below the threshold, the total ICP-MS integrated intensity increased slowly with laser irradiance on a log-log scale. Beyond the threshold, the slope was much greater. Similar behaviors 
were observed for laser beam spot sizes of 35, 55 and $65 \mu \mathrm{m}$. In general, a larger beam size yields a higher integrated signal intensity due to more mass removed from a larger area. Even though the growth of the crater depth and net volume (the volume below the original surface subtracted by the volume above) rolls off above $200 \mathrm{GW} / \mathrm{cm}^{2}$ in figure 6 , the ICP-MS signal continually increases above this irradiance. Despite the roll-off of the total mass ablated from the sample, more mass is entrained and transported to the ICP at the higher irradiances. The reason for this trend is not yet fully understood; some possible explanations are discussed in following section.

\section{d) Entrainment and transport efficiency dependence on laser irradiance}

The ablated particle size distribution changes once phase explosion occurs; particulates larger than 10 micron were observed above the phase explosion threshold (c.f. figure 3). Large particulates will not be entrained into the argon gas flow and will fall into the ablation chamber and in the transport tubing due to gravity ${ }^{14}$. Thus, in the irradiance range where phase explosion occurs, entrainment and transport to the ICP should decrease. To assess the effect of phase explosion on particulate entrainment and transport efficiency, the ratio of the net ICP-MS intensity (integrated counts with background subtraction) to the net crater volume was measured for laser irradiance from $5 \times 10^{9}$ to $1 \times 10^{12} \mathrm{~W} / \mathrm{cm}^{2}$ for several beam spot sizes (figure 7). The ratio decreased gradually up to the phase explosion threshold irradiance, stays flat at a minimum value, and then rises again at $\sim 100 \mathrm{GW} / \mathrm{cm}^{2}$. The observed decrease in the ratio for increasing irradiance below the threshold is consistent with previous data by Jeong et al using glass 
samples ${ }^{14}$. The decrease is likely due to the increase in the number of larger particulates. Once phase explosion occurs, the ratio (transport efficiency) remained at its lowest value.

The fact that the ratio increases well above the phase explosion threshold is interesting. At this irradiance region, the crater depth rolled off, but the ICP-MS signal continually increased. The reason is unclear. One possibility is that at higher irradiance, strong absorption of the incident laser light by the plasma could create a high temperature and pressure region through which the particulates must traverse. The large particulates, upon interacting with the hot dense plasma, can be broken into smaller size particulates that will be better entrained and transported.

\section{CONCLUSION}

This work has demonstrated the existence of phase explosion and its influence on ICP-MS signal intensity. The threshold irradiance for phase explosion has dependence on both laser wavelength and beam spot size; higher threshold irradiance and smaller beam size were required for longer wavelengths to induce phase explosion. Although entrainment and transport efficiency were minimal at the phase explosion threshold, they increased substantially above the threshold. In this irradiance range, the hot and dense plasma layer may be effective in breaking the large particulates into smaller sizes that are readily entrained and transported.

Using high irradiance above the phase explosion threshold may have several benefits for laser solid sampling. A composite sample material can be heated instantaneously and a pool of superheated liquid, comprised of all elements above the boiling point, may form. This rapid heating of the sample to a temperature near the critical state may eliminate factors such as preferential vaporization and elemental 
fractionation, leading to good stoichiometric representation of ablated mass. Phase explosion was not only observed in single crystal silicon, but also in other metal and glass samples. Another reason for looking the phase explosion region is to determine if we can identify a material independent region; i.e. an irradiance region where the quantity of mass ablated is independent on material properties. Such work is ongoing and will be reported in the near future.

\section{ACKNOWLEDGEMENT}

This research was supported by the U. S. Department of Energy, Office of Basic Energy Sciences, Chemical Sciences Division, Chemical Separations and Analysis Branch, and by the Office of Nonproliferation and National Security (NN20) under Contract No. DE-AC03-76SF00098. 


\section{REFERENCE}

1. Russo, R.E.; Mao, X.L.; Borisov, O.V.; Trends in Anal. Chem. 1998, 17, 461-469.

2. Gunther, D.; Horn, I.; Hattendorf, B.; Fresenius J. Anal. Chem. 2000, 368, 4-14.

3. Liu, X. R; Horlick G. Spectrochim. Acta 1995, 50B 537-548.

4. Winefordner, J.D.; Gornushkin, I.B.; Pappas, D.; Martveev, O.I.; Smith, B.W. J. anal. At. Spectrum. 2000, 15, 1161-1189.

5. Leach, J.J.; Allen, L.A.; Aeschliman, D.B.; Houk, R.S. Anal. Chem. 1999, 71, 440445.

6. Moenke-Blankenburg, L. Spectrochim. Acta Rev. 1993, 15,1-37

7. Darke, S.A.; and Tyson, J.F. J. Anal. At. Spectrom. 1993, 8, 145-209

8. Outridge; P.M.; Doherty, W.; Gregoire, D.C. Spectrochim. Acta, 1997, 52B, 20932102

9. Arrowsmith, P. Anal. Chem. 1987 59, 1437-1444

10. Denoyer, E.R.; Fredeen, K.J.; and Hager, J.W. Anal. Chem. 1991, 63, A445-A457

11. Hemmerlin, M.; Somas, D.; Dubuisson, C.; Loisy, F.; Poussel, E.; Mermet, J.M. Fresenius J. Anal. Chem. 2000, 368, 31-36.

12. Cromwell, E.F. Arrowsmith, P. Anal. Chem. 1995, 67, 131-138

13. Chan, W.T.; Mao, X.L.; Russo, R.E. Appl. Spectrosc. 1992, 46, 1025-1031

14. Jeong, S.H.; Borisov, O.V.; Yoo, J.H.; Mao, X.L.; Russo, R.E.: Anal. Chem. 1999, $71,5123-5130$

15. Mao, X.L.; Ciocan, A.C.; Russo, R.E. Appl. Spectrosc., 1998, 52, 913-918

16. Shannon, M. A.; Mao, X.L.; Fernandez, A.; Chan, W.T.; and Russo, R. E. Anal. Chem. 1995, 67, 4522-4529 
17. Yoo, J.H.; Jeong, S.H.; Mao, X.L.; Greif, R.; Russo, R.E. Appl. Phys. Lett., 2000, 76 $783-785$

18. Yoo, J.H.; Jeong, S.H.; Greif, R.; Russo, R.E J. OF Appl. Phys., 2000, 88, 1638-1649

19. Martynyuk, M.M.: Sov. Phys. Tech. Phys. 1976, 21, 430

20. Phipps C. R.; and Dreyfus R.W.; in Laser Ionization Mass Analysis, Vertes A.; Gijbels R.; and Adams F. (Eds), John Wiley \& Son, New York, 1993, Chapter

21. Phipps C. R.; Jr, Turner Y.P.; Harrison R. F.; York G.W.; Osborne W.Z.; Anderson G.K.; Corlis X.F.; Haynes L.C.; Steele H.S.; and Spicochi K.C., J. Appl. Phys., 1988, 64, 1083.

22. Liu, H.C.; Mao, X.L.; Yoo, J.H.; Russo, R.E. Spectrochim. Acta 1999, 54B, 16071624

23. Balazs L.; Gijbels R.; and Vertes A., Analyt. Chem., 1991, 63, 314-320.

24. Schittenhelm, H.; Callies, G.; Straub, A.; Berger, P.; Hugel, H. J. Phys. D: Appl. Phys. 1996, 29, 1564-1575

25. Breitling, D.; Schittenhelm, H.; Berger, P.; Dausinger, F.; Hugel, H. Appy. Phys. A 2000, 69, S505-S508

26. Mao, X.L.; Russo, R.E Appl. Phys. A, 1997, 64, 1-6

27. Eyett, M.; and Bäuerle, D.; Appl. Phys. Lett., 1987, 51, 2054-2055.

28. Shannon, M. A.; Mao, X. L.; Fernandez, A.; Chan, W.T.; Russo, R. E. Anal. Chem. 1995, $67,4522-4529$

29. Fernandez, A.; Mao, X. L.; Chan, W.T.; Shannon, M. A.; Russo, R. E. Anal. Chem. $1995,67,2444-2450$ 


\section{FIGURE CAPTION:}

Figure 1. Laser shadowgraph imaging experimental setup. The pump beam is a 266 $\mathrm{nm}, 3 \mathrm{~ns} \mathrm{Nd}$ :YAG laser beam. The probe beam is a $532 \mathrm{~nm}, 35 \mathrm{ps}$ Nd:YAG laser beam. A detail of shadowgraph setup was insert on the top left of the graph.

Figure 2. Cross sectional images of the crater for a) laser irradiance slightly below the phase explosion threshold $\left(20 \mathrm{GW} / \mathrm{cm}^{2}\right)$ and b) laser irradiance slightly above the threshold $\left(24 \mathrm{GW} / \mathrm{cm}^{2}\right)$. The focused laser spot was $35 \mu \mathrm{m}$ and the phase explosion threshold was at $22 \mathrm{GW} / \mathrm{cm}^{2}$.

Figure 3. Sequence of mass ejection images obtained by laser shadowgraphy for the laser irradiance of $3.9 \times 10^{10} \mathrm{~W} / \mathrm{cm}^{2}$.

Figure 4. Dependence of crater depth on incident wavelength for laser beam spot size of $35 \mu \mathrm{m}$.

Figure 5. Dependence of phase explosion threshold on laser beam sizes at $\lambda=532$ $\mathrm{nm}$ and $266 \mathrm{~nm}$.

Figure 6. Correlation of ICP-MS signal with the crater depth for high irradiance laser ablation of silicon involving phase explosion.

Figure 7. Ratio of ICP-MS intensity to crater volume as a function of laser irradiance. 